\title{
Acknowledgment of Reviewers, 2016
}

The success of Phytopathology depends on the quality of manuscripts submitted by authors and on the care and competence with which they are reviewed. It is the policy of the Editorial Board to solicit reviews for manuscripts from specialists who are most qualified to review them. In addition to members of the Editorial Board, the individuals listed below provide constructive critical reviews of one or more manuscripts during the past year. Their names are published here in grateful appreciation of their contribution to the journal and to the science of phytopathology. http://dx.doi.org/10.1094/PHYTO-107-1-0004

Nilwala Abeysekara

Maricelis Acevedo

Srđan Aćimović

Karen Aitken

Eduard Akhunov

Maher Al Rwahnih

Olufemi Alabi

Sandra Alaniz

Akhtar Ali

Gul Ali

Tom Allen

Rodrigo Almeida

Achour Amiri

El-Desouky Ammar

Diaoguo An

Didier Andrivon

Janis Antonovics

Gavin Ash

Davoud Ashourloo

Jacques Avelino

Ofir Bahar

Brian Bahder

Bochra Bahri

Hu Baishi

Mounes Bakhshi

Matthew Bakker

Guus Bakkeren

George Baley

Peter Balint-Kurti

David Baltrus

Marie-Odile Bancal

Sabine Banniza

Urmil Bansal

Paola Barba

John J. Beck

Janna Beckerman

Dominik Begerow

Eduardo Bejarano

Alessandra Belisario

Roeland Berendsen

Gabriele Berg

Anna Berlin

Assunta Bertaccini

Lien Bertier

Subhash Bhardwaj

Dawn Bignell

Guillaume Bilodeau

A. Nicholas E. Birch

Burton Bluhm

Clive Bock

Melvin Bolton

John Bonman

Domenico Bosco

Robert Bowden

Joanna K. Bowen

Uwe Braun

Marin Brewer

Robert Briddon

Robert Brueggeman

J. Bruenn

Glenn Bryan

Hermann Buerstmayr
Russ Bulluck

Roberto Buonaurio

Lindsey Burbank

Margi I. Butler

Hadi Bux

Emmanuel Byamukama

Santa Olga Cacciola

Lance Cadle-Davidson

Guohong Cai

Thierry Candresse

Liliana Cano

Massimiliano Cardinale

Odile Carisse

Angus J. Carnegie

John Carr

Sergio Casas-Flores

Clare Casteel

Vanina Castroagudin

Filipe Catry

Francisco Cazorla

Paulo Ceresini

Daniel Chapman

Amy Charkowski

Subhadeep Chatterjee

Dan Chellemi

Charles Chen

Hongxin Chen

Jianchi Chen

Jianli Chen

Weidong Chen

Xianming Chen

Yu Chen

L. Chernin

Jed Christianson

Chia-Lin Chung

Christopher A. Clark

Will Cody

Helvécio Coletta-Filho

Stephane Compant

Richard Cooper

Teresa Coutinho

Christina Cowger

Kerik Cox

Maria Cristina Lura

Will Cuddy

Albert Culbreath

Christian Cumagun

Nik Cunniffe

Kent Daane

Reza Darvishzadeh

Lawrence Datnoff

Jenny Davidson

Mike Davis

Richard Davis

Antonieta De Cal

Leo de la Fuente

Luiz de Oliveira

Gezimar D. de Souza

Antonio de Vicente Moreno

Erick De Wolf

M. W. Dees

Emerson Del Ponte
Luis del Rio

Régine Delourme

Mike Deom

Megan Dewdney

Juan Antonio Díaz-Pendón

Brian Diers

Savithramma Dinesh-Kumar

Shefali Dobhal

Aviv Dombrovsky

Leslie Domier

Nicole Donofrio

Max Dow

Tyler Dreaden

Andre Drenth

Martin Drucker

Ian Dry

Nicholas Dufault

Frank Dugan

Jeremiah Dung

Tuan A. Duong

Yigal Elad

Monica Elliott

Jeff Ellis

Larry Englander

Armin Erlacher

Paul Esker

Sydney Everhart

Ahmad Fakhoury

Adam Famoso

Mark Farman

John Fellers

Dilantha Fernando

Inmaculada Ferriol

Tom Fetch

Sabine Fillinger

Svetlana Folimonova

Greg Forbes

Gerda Fourie

Bart Fraaije

Emilie Fradin

Kenneth Frost

William Fry

Marc Fuchs

N. Fukino

Joe Funderburk

Giorgio Gambino

Abraham Gamliel

Maria Garcia-Pedrajas

Angelo Garibaldi

David Gent

A. S. Gesteira

Amanda Gevens

Said Ghabrial

Godelieve Gheysen

Mark Gijzen

Robert Gilbertson

Karl Glover

Scott Gold

Maria Teresa Gonzalez-Jaen

Celedonio Gonzalez

Steve Goodwin

Tom Gordon
Erica Goss

Rubella Goswami

Manje Gowda

Jim H. Graham

Stewart Gray

Rita Grosch

Aslihan Gunel

Liyun Guo

A. Gutermuth

Osman Gutierrez

Ahmed Hadidi

Susan Halbert

Bernardo de Almeida HalfeldVieira

Dennis Halterman

Jong Hyun Ham

Mohamed Sobhy Hamada

Richard Hamelin

Kim Hammond-Kosack

John Hammond

Rosemarie Hammond

Jianjun J. Hao

W. Hao

Philip Harmon

Scott Harper

John Hartung

Nichola Hawkins

Johannes Helder

Yael Helman

Joe Helps

Flávio Henrique-Silva

Colin Hiebert

Mark Hilf

Brad Hillman

David Hodson

Monica Höfte

Donald Hopkins

Y. Huang

Iori Imazaki

Patrik Inderbitzin

Renaud Ioos

Rob Jackson

Jonathan M. Jacobs

Chatchawan Jantasuriyarat

Marie-Agnès Jaques

Michael Jeger

Kshirod Jena

Jong-Seong Jeon

Pingsheng Ji

María del Mar Jiménez-Gasco

Young-Ki Jo

Dennis Johnson

Ken Johnson

Eirian Jones

Jeffrey Jones

John Jones

Richard Jones

Howard Judelson

Thomas Jung

Wayne Jurick II

Parijat Juvale

Mehdi Kabbage 
Byung-Ho Kang

Seogchan Kang

George Karaoglanidis

Alexander Karasev

Jaacov Katan

Anthony Keinath

Mohamed Khan

Nabil Killiny

Kwang-Hyung Kim

Yong-Ki (Richard) Kim

Levente Kiss

Anna Klimes

Steven Klosterman

Alissa Kriss

Rodrigo Krugner

Masaharu Kubota

Sridhara Kunjeti

Kris Lambert

Blanca Landa

Alessandra Lanubile

Nicholas Larkan

Robert Larkin

Conor Lawless

Christophe Le May

Seonghee Lee

Anne Legrève

Kathy Lewis

Zenglu Li

Ziqin Li

Yu-Cai Liao

Morten Lillemo

Celeste Linde

Magdalen Lindeberg

Steven Lindow

Chunji Liu

Xi Liu

Juan Jose Lopez-Moya

S. Loreti

J. Ludwig-Mueller

Chao-Xi Luo

Yong Luo

Zhonghua Ma

Stuart MacFarlane

Laurence Madden

Walt Mahaffee

Subas Malla

Kranthi Mandadi

Patricia Manosalva

Benoit Marçais

Hans Maree

Sasha Marine

Sam Markell

Abi S. A. Marques

Robert Martin

R. Mason

Alex Mastin

Janet Matanguihan

Diane Mather

Febina Mathew

Gladys Mbofung

Margaret McGrath

Leah McHale

Robert McIntosh

Lucky Mehra

Ulrich Melcher

Terence Mhora

Santiago Mideros

Thomas Miedaner

Michael Milgroom

Svetlana Milijašević-Marčić

J. Creighton Miller

Alice Milne
Zhou Mingguo

Thomas Mitchell

Peter Moffett

V. Mohler

Daniel Molitor

Enrique Moriones

Matt Moscou

Gary Muehlbauer

Chris Mundt

Gary Munkvold

Joseph Munyaneza

Brian Nault

Juan Navas-Cortés

Rebecca Nelson

Scot Nelson

James Ng

Terry Niblack

Rients Niks

Chenxing Niu

Berit Nordskog

Leonard Nunney

P. D. O'Boyle

Kerry O'Donnell

Jamie O'Rourke

Erich Christian Oerke

Peter Ojiambo

Patricia Okubara

Rabiu Olatinwo

Ely Oliveira García

Richard Oliver

Peter Oudemans

Franck Panabières

Kalliope Papadopoulou

Julien Papaix

Hanu Pappu

Stephen Parnell

Russell Paterson

Timothy Paulitz

Tobin Peever

Natalia Peres

Giancarlo Perrone

Keith Perry

Kari Peter

Sarah Pethybridge

Benjamin Petre

Gerhard Pietersen

John Pitkin

Randy Ploetz

Luigi Ponti

Mikhail Pooggin

Neha Potnis

Patil Prabhu

Sean Prager

Ankush Prashar

Philippe Prior

Dov Prusky

Olivier Pruvost

Alexander Putman

Wenping Qiu

Feng Qu

Lina Quesada-Ocampo

Tod Ramsfield

Margaret Redinbaugh

Sebastien Rigali

Barbara Robbertse

Liliana Rocha

Marion Röder

Megan Romberg

Vittorio Rossi

Dorith Rotenberg

Mike Rott

Matthew Rouse
David Rousseau

Thierry Rouxel

Adib Rowhani

Michelina Ruocco

Daria Rybakova

Erika Saalau Rojas

Ivan Sache

Seiya Saito

Berit Samils

Soum Sanogo

Maria Saponari

Serge Savary

Kyrylo G. Savchenko

Leonardo Schena

Harald Scherm

Annemiek Schilder

David A. Schisler

Daniel Schlatter

Bernd Schneider

William Schneider

Corine Schoebel

James Schoelz

Herman Scholthof

Nathan Schroeder

Kristin Schulz

Marco Scortichini

Jason Scott

Endang Septiningsih

Ramos Sepulveda

Jyoti Shah

Weixing Shan

Amir Sharon

Michael Shaw

Kai Shi

Nina Shishkoff

Dylan Short

Cristina Silvar

A. M. Simmons

Mark Sisterson

Pete Skelsey

Jadwiga Śliwka

Grant Smith

Baruch Sneh

Teruo Sone

Jose Soriano

Rajagopalbab Srinivasan

Horst-Henning Steinmann

Drake Stenger

Lukasz Stepien

Ioannis Stergiopoulos

Lucy Stewart

Jinita Sthapit Kandel

Benjamin Stich

Ed Stover

Stephen Strelkov

David Studholme

Mysore Sudarshana

Ramesh Sundar

George Sundin

Garry Sunter

Les Szabo

Susumu Takamatsu

Pedro Talhinhas

Pedro Talhinhas

Satyanarayana Tatineni

Louise F. Thatcher

Marco Thines

Howard Thomas

Linda Thomashow

Jeremy Thompson

Miaoying Tian

Sotirios E. Tjamos
Maria Tomaso-Peterson

Lesley Torrance

Gabriel Torres

Lindsay Triplett

Mariette Truter

William Turechek

Massimo Turina

Ioannis Tzanetakis

Jose Urbez-Torres

Toshiyuki Usami

Lisa Vaillancourt

Jari Valkonen

Femke van den Berg

Aletta van der Merwe

Rene Van der Vlugt

Wopke van der Werf

Anne van Diepeningen

Joel Vanneste

M. Dolores Vazquez

Vittorio Venturi

Jeanmarie Verchot-Lubicz

Anne Vidaver

Paolo Villa

Boris Vinatzer

David Walker

Nathan Walker

Guo-Liang Wang

Jianying Wang

Lin Wang

Nian Wang

Marilyn Warburton

Kazuo Watanabe

Astri Wayadande

Kimberly Webb

Melanie Weckert

Bevan Weir

Laure Weisskopf

Jon West

Andreas Westphal

Phillip Wharton

Terry Wheeler

Ross Whetten

Steve Whitham

Barny Whitman

Emmanuel Wicker

Timothy Widmer

Edward P. Wilhelm

Laetitia Willocquet

Katelyn Willyerd

Calum Wilson

Richard Wilson

Stephan Winter

William Wintermantel

Bo Ming Wu

Jingqin $\mathrm{Wu}$

Jiatao Xie

Mingliang Xu

Xiangming $\mathrm{Xu}$

Yangchun $\mathrm{Xu}$

Yong $\mathrm{Xu}$

Mikihiro Yamamoto

Jennifer Yates

Iris Yedidia

Y. N. Yin

Nevin Young

Jonathan Yuen

Murilo Zerbini

Jianfu Zhang

Youfu Zhao

Shaobin Zhong 